\title{
Calcul en éléments finis des déformations des sols non saturés : équations et exemples
}

\section{WONE}

J.-P. MAGNAN

Laboratoire Central des Ponts et Chaussées 58 , boulevard Lefebvre 75732 Paris Cedex 15

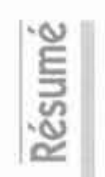

Cet article présente un modèle numérique en éléments finis construit sur la base des équations de la consolidation des sols élastoplastiques non saturés. Ce modèle a été programmé dans le code de calcul CESARLCPC.

Les équations du modèle tiennent compte de l'interaction des déformations du squelette, des écoulements de l'eau et de l'air ainsi que des mouvements de l'air dissous dans l'eau, La résolution numérique de ces équations associe la méthode des éléments finis pour discrétiser l'espace et un schéma d'intégration implicite pour discrétiser le temps. Les déformations du squelette sont décrites selon l'approche des variables indépendantes.

Deux applications sont décrites, à un tube épais en condition axisymétrique et à un massif de sol en déformation plane.

\section{Finite element analysis of the deformations of unsaturated soils: equations and exemples}

This paper describes a finite element numerical model derived from the basic equations of elastoplasticity and consolidation of unsaturated soils. The model was implemented in the finite element program CESAR-LCPC.

The equations of the model aceount for the interaction of the deformation of the soil skeleton, of the flow of water and air and of the movement of the air dissolved in the pore water. The numerical solution of these equations is based on the finite element method for space discretisation and on an implicit method for time discretisation. The deformations of the soil skeleton are described within the framework of the independent variable approach.

Two applications of this model to an axisymmetric problem (thick tube) and to a plane strain problem (2-D consolidation of a soil mass) are presented. 


\section{Introduction}

La modélisation du comportement mécanique des sols non saturés ne fait pas l'objet d'un consensus parmi les spécialistes de la géotechnique. L'extension de la notion de contraintes effectives, telle qu'elle a été proposée par Bishop (1961) pour servir de cadre à la description des sols, a fait l'objet de différentes critiques, souvent étayées par des résultats expérimentaux obtenus en laboratoire (Jennings et Burland, 1962 ; Matyas et Radakrishna, 1968; Fredlund et Morgenstern, 1976). D'autres approches, comme celles d'Alonso et al. (1988, 1990), ou de Fredlund (1989) semblent apporter une réponse plus complète et mieux décrire la réalité par une modélisation physique avec deux champs de contraintes indépendants.

Différents modèles théoriques et numériques basés sur les modèles physiques en contraintes effectives ou en variables indépendantes sont apparus depuis une dizaine d'années, comme ceux d'Alonso et al. (1988), de Nanda (1989), de Gens et al. (1995) et de Gatmiri et al. (1995).

L'étude présentée dans cet article a été réalisée au moyen du code de calcul en éléments finis CESARLCPC et a cherché à appliquer à la programmation des équations des sols non saturés la même démarche systématique que pour les calculs relatifs aux sols saturés. Pour décrire le comportement des sols non saturés, nous avons utilisé une formulation couplée et un comportement élastoplastique avec écrouissage du squelette du sol, qui permet de prendre en compte l'irréversibilité des déformations.

Cet article rappelle les équations qui représentent le comportement élémentaire des sols non saturés, décrit les étapes de leur transformation en équations matricielles adaptées à leur résolution par la méthode des éléments finis, puis présente quelques résultats de calcul.

\section{2}

\section{Hypothèses et définitions}

La représentation mêcanique que nous avons adoptée pour la modélisation numérique des sols non saturés repose sur la superposition de trois milieux continus, qui couvrent chacun l'ensemble de l'espace occupé par le sol :

- le milieu global (sans distinguer de phases ; on admet que les modifications de la composition de ce milieu triphasé par suite des mouvements de l'eau et de l'air ont un effet négligeable sur ses propriétés physiques et mécaniques, c'est-à-dire que l'on peut définir une loi de comportement à partir de son état initial) :

- le milieu continu « air » et le milieu continu « eau » (ces deux milieux peuvent se déplacer l'un par rapport à l'autre à l'intérieur du milieu global et en sortir ou y entrer).

\section{1}

\section{Champs de contraintes}

Trois champs de contraintes sont définis respectivement dans le milieu global, l'eau et l'air : la contrainte totale $\sigma$, la pression de l'eau $p_{w}$ et la pression de l'air $p_{\mathrm{a}}$.
Les conventions de signe utilisées dans les équations sont des conventions « mixtes » : contraintes totales négatives en compression, pressions d'eau et d'air positives en compression.

Pour le calcul, les pressions d'eau et d'air sont remplacées par les charges d'eau $h_{w}$ et d'air $h_{i}$ définies, selon les habitudes de la mécanique des sols, par:

$$
h_{w}=\frac{P_{w}}{\gamma_{w}}+z \quad \text { et } \quad h_{a}=\frac{P_{b}}{\gamma_{a}}+z
$$

avec:

$\gamma_{w}$ : poids volumique de l'eau ;

$\gamma_{a}$ : poids volumique de l'air ;

$\mathrm{Z}$ : altitude par rapport à un repère fixe.

Le champ de contraintes du milieu global dépend directement de la pesanteur et des conditions aux limites et, indirectement, de la loi de comportement et des autres conditions aux limites. Le champ associé à la pression de Yeau (respectivement, à la pression de l'air) dépend directement de la pesanteur et des conditions aux limites sur la charge d'eau (respectivement, la charge de l'air) et, indirectement, de la loi de comportement du matériau global et des autres conditions aux limites.

\section{2}

\section{Champs de déplacements et de déformations}

Trois champs de déplacements, auxquels sont associés trois champs de déformations, sont utilisés pour décrire l'état du sol non saturé :

- un champ de déplacements associé au milieu continu global ou « champ principal », noté $\overrightarrow{\mathrm{u}}$;

- un champ de déplacements associé à l'eau, noté $\overrightarrow{\mathrm{u}}_{\mathrm{w}}$; - un champ de déplacements associé à lair, noté $\overrightarrow{\mathrm{u}}_{\mathrm{a}}$.

Au champ de déplacements principal $\vec{u}$ est associé le champ de déformations totales du milieu global $\varepsilon$. Le champ de déplacements associé à l'eau est utilisé sous la forme du vecteur des vitesses moyennes d'écoulement de l'eau $\vec{v}_{w}$ et sous la forme d'un scalaire égal au flux d'eau sortant d'un volume unitaire, div $\vec{v}_{w}$ (et de même pour les déplacements associés à l'air : $\vec{v}_{\mathrm{a}}$ et $\operatorname{div} \overrightarrow{\mathrm{v}}_{\mathrm{a}}$ ).

Les déformations totales $\varepsilon$ sont négatives en contraction, et les flux sont positifs quand ils sortent du volume élémentaire.

\section{3}

\section{Comportement mécanique}

On suppose que les déformations du milieu peuvent être induites par une variation de la contrainte totale $\left(\sigma+p_{\mathrm{a}}\right)$ et/ou de la succion $\left(\mathrm{p}_{\mathrm{a}}-\mathrm{p}_{\mathrm{w}}\right)$, considérées comme variables indépendantes. La loi de comportement est de type élastoplastique avec écrouissage. Elle est décrite dans cet article avec les équations proposées par Alonso et al. (1990), mais peut être transformée aisément pour accueillir d'autres formes de lois élastoplastiques avec ou sans écrouissage.

Les contraintes et les déformations doivent satisfaire simultanément les équations d'équilibre et la loi de comportement. 


\section{Équations d'équilibre}

$\sigma_{i, j}+F_{1}=0$

avec:

$\sigma_{i 1}$ : tenseur des contraintes totales :

$\mathrm{F}_{1}$ : forces volumiques.

\section{2}

\section{Loi de comportement du milieu global (Alonso et al., 1990)}

Cette loi de comportement est écrite sous la forme incrémentale :

avec:

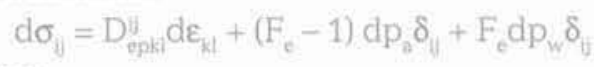

$\mathrm{D}_{\text {ep }}$ : matrice de souplesse du milieu global relative aux variations de $\sigma_{i \mid}+p_{a} \delta_{i \mid}$ :

$\mathrm{d} \varepsilon_{\mathrm{k} \mid}$ : incrément du tenseur des déformations (du milieu global) ;

$F_{e}=D_{e p} D_{s}$;

$D_{s}$ : matrice de souplesse du milieu global relative aux variations de $\left(\mathrm{p}_{\mathrm{a}}-\mathrm{p}_{\mathrm{w}}\right)$ :

$\delta_{\mathrm{i}}$ : symbole de Kronecker ;

$\mathrm{dp}_{\mathrm{a}}$ : incrément de pression d'air :

$\mathrm{dp}_{\mathrm{w}}$ : incrément de pression d'eau.

\section{4}

\section{Écoulements de l'eau et de l'air dans le milieu poreux}

L'eau et l'air se déplacent dans l'espace occupé par le milieu global en respectant, d'une part, des lois de conservation de la masse et, d'autre part, des lois reliant les vitesses moyennes d'écoulement aux gradients de charge (loi de Darcy pour chaque phase). Ces vitesses moyennes désignent les vitesses relatives des fluides par rapport au solide, comme si tout l'espace (solide+pores) leur était offert.

\section{1}

\section{Équation de conservation de la masse d'eau}

$$
\frac{\partial}{\partial t}\left(\rho_{w} n S_{r}\right)+\operatorname{div}\left(\rho_{w} \vec{v}_{w}\right)=0
$$

avec :

$\rho_{\mathrm{w}}$ : masse volumique de l'eau;

$n$ : porosité du massif; ;

$\overrightarrow{\mathrm{V}}_{\mathrm{w}}$ : vitesse moyenne d'écoulement de l'eau ;

$\mathrm{S}_{\text {, }}$ degré de saturation en eau, qui dépend des pressions d'eau et d'air et de $\underline{\underline{\sigma}}$. On a adopté pour décrire cette dépendance la relation utilisée par Matyas et Radakrishna (1968) et reprise par Alonso et al. (1988) :

$$
\mathrm{S}_{\mathrm{r}}=\mathrm{S}_{\mathrm{ro}}-\left[\mathrm{a}_{\mathrm{s}}+\mathrm{b}_{\mathrm{s}}\left(\sigma_{\mathrm{ij}}+\mathrm{p}_{\mathrm{a}} \delta_{\mathrm{ij}}\right)\right]\left\{1-\exp \left[-\mathrm{c}_{\mathrm{s}}\left(\mathrm{p}_{\mathrm{a}}-\mathrm{p}_{\mathrm{w}}\right)\right]\right\}
$$

avec :

$\mathrm{S}_{\mathrm{r0}}$ : degré de saturation initial :

$\mathrm{a}_{\mathrm{s}}, \mathrm{b}_{\mathrm{s}^{\prime}} \mathrm{c}_{\mathrm{s}}$ : constantes.

\section{Équation de conservation de la masse d'air}

$$
\frac{\partial}{\partial t}\left[\rho_{\mathrm{a}} \mathrm{n}\left(1-\mathrm{S}_{\mathrm{r}}+H \mathrm{~S}_{\mathrm{r}}\right)\right]+\operatorname{div}\left[\rho_{\mathrm{a}}\left(\overrightarrow{\mathrm{v}}_{\mathrm{a}}+\mathrm{H} \vec{v}_{\mathrm{w}}\right)\right]=0
$$

avec:

$p_{\mathrm{a}}$ : masse volumique de l'air :

$\mathrm{H}$ : coefficient de solubilité de l'air dans l'eau ou coefficient de Henry $(\mathrm{H}=0,02)$;

$\vec{v}_{a}$ : vitesse moyenne d'écoulement de l'air.

\section{3}

\section{Loi de Darcy pour l'eau}

La loi de Darcy s'écrit:

$$
\overrightarrow{\mathrm{v}}_{\mathrm{w}}=\underline{\underline{\underline{\mathrm{k}_{\mathrm{w}}}}} \operatorname{gra} \overrightarrow{\mathrm{d}} \mathrm{h}_{\mathrm{w}}
$$

avec :

$h_{\text {. }}$ : charge hydraulique;

$\underline{\underline{k_{w}}}$ : tenseur des coefficients de perméabilité à l'eau (les coefficients de perméabilité dépendent de façon générale de l'indice des vides ou de la porosité, du degré de saturation, de la température et de la nature du fluide).

La fonction adoptée pour décrire les variations des coefficients de perméabilité à l'eau est la même que celle utilisée par Alonso et al. (1988), Nanda (1989) et Abida (1992) :

$$
\mathrm{k}_{\mathrm{w}}=\mathrm{a} 10^{\alpha e}\left[\frac{\mathrm{S}_{\mathrm{r}}-\mathrm{S}_{\mathrm{ru}}}{1-\mathrm{S}_{\mathrm{ru}}}\right]^{3}
$$

avec :

a, $\alpha$ : constantes ;

$\mathrm{e}$ : indice des vides;

$\mathrm{S}_{r}$ : degré de saturation :

$\mathrm{S}_{\mathrm{n}}$ : degré de saturation résiduel.

\section{4}

\section{Loi de Darcy pour l'air}

On admet que l'écoulement de l'air est également régi par la loi de Darcy (Yoshimi, 1969) :

$$
\overrightarrow{\mathrm{v}}_{\mathrm{a}}=-\underline{\underline{\mathrm{k}_{\mathrm{a}}}} \mathrm{gra \textrm {d }} \mathrm{h}_{\mathrm{a}}
$$

avec $: \underline{\underline{k_{\mathrm{a}}}}$ : tenseur des coefficients de perméabilité à l'air.

La fonction décrivant les variations des coefficients de perméabilité à l'air est celle utilisée par Alonso et al. (1988), Nanda (1989) et Abida (1992) :

$$
\mathrm{k}_{\mathrm{a}}=\mathrm{b} \frac{\gamma_{\mathrm{a}}}{\mu_{\mathrm{a}}}\left[\mathrm{e}\left(1-\mathrm{S}_{\mathrm{r}}\right)\right]^{\mathrm{c}}
$$

avec :

b, c : constantes adimensionnelles :

$\gamma_{0}$ : poids volumique de l'air ;

$\mu_{4}$ : viscosité de l'air ;

$\mathrm{e}$ : indice des vides. 


\section{Conditions aux limites}

Les conditions aux limites imposées aux inconnues (déplacements et pressions) sont des déplacements ou forces imposés au milieu global, des flux ou des charges d'eau imposés pour l'eau et des flux ou des charges d'air imposés pour l'air.

\section{6}

\section{Conditions initiales}

Dans les calculs de comportement des milieux poreux non saturés, il est souvent difficile d'imaginer l'état initial. En effet, les distributions des contraintes totales, des charges d'eau et des charges d'air doivent être en équilibre compte tenu de la répartition des phases dans l'espace (indice des vides et degré de saturation), si l'on veut que les résultats des calculs ne combinent pas l'effet de l'évolution spontanée de l'état initial et l'effet du chargement.

Pour définir un état initial en équilibre, nous avons appliqué une procédure qui consiste à calculer l'état initial de la manière suivante :

- on effectue à partir d'un état initial estimé mais pas nécessairement en équilibre un premier calcul avec pour seul chargement mécanique le poids du massif de sol, jusqu'à stabilisation des charges d'eau et d'air et des contraintes;

- on prend les résultats de ce calcul comme état initial des calculs ultérieurs en annulant les déplacements.

\section{7}

\section{Choix d'un principe variationnel}

Le passage des équations prẻcédentes à une formulation variationnelle permettant d'associer la solution cherchée (un état d'équilibre local) à un extrémum d'une fonctionnelle (minimum ou maximum) n'est pas une opération classique dans le cas des milieux triphasiques.

La procédure que nous avons adoptée comporte deux étapes :

- Dans la première étape, on fige l'état des fluides et on se préoccupe de trouver l'état d'équilibre du milieu global en minimisant son énergie potentielle, c'est-à-dire que l'on écrit un " principe des travaux virtuels m dans lequel le travail interne (déduit de la loi de comportement) est équilibré par le travail des forces externes (forces de pesanteur et forces de surfaces, c'est-à-dire pressions totales) :

$$
\int_{\Omega} \sigma \delta \varepsilon_{j} \mathrm{~d} \Omega-\int_{\Omega} \mathrm{F} \delta u_{1} \mathrm{~d} \Omega-\int_{S_{\sigma}} \mathrm{T}_{\mathrm{j}} \delta \mathrm{u}_{\mathrm{j}} \mathrm{dS} \mathrm{S}_{\mathrm{a}}=0
$$

Cette intégrale correspond au travail de la contrainte totale. Le champ de contrainte $\sigma$ est défini sur le milieu global et correspond à $\varepsilon$.

- Dans la seconde étape, on s'occupe des équations d'écoulement et on écrit des équations correspondant aux bilans globaux des mouvements des masses fluides (eau et air) présentes dans le sol, compte tenu de l'écoulement, de la compressibilité des fluides, des variations du degré de saturation et des variations des volumes des pores:
- pour l'eau

$\int_{\Omega} a_{w} n S_{r} \dot{p}_{w} \delta p_{w} d \Omega+\int_{\Omega} p_{w} n \dot{S}_{r} \delta p_{w} d \Omega-\int_{\Omega} \rho_{w} S_{r} \delta_{j j} \dot{\varepsilon}_{j u} \delta p_{w} d \Omega-$ $\int_{\Omega} \operatorname{div}\left[\rho_{w} k_{w} \operatorname{grad}\left(\frac{P_{w}}{\gamma_{w}}+z\right)\right] \delta p_{w} d \Omega+\int_{S_{\phi_{w}}} \phi_{w} \delta p_{w} d S_{\phi_{w}}=0$ - pour l'air:

$\int_{\Omega} a_{\mathrm{a}} \mathrm{n}\left(1-\mathrm{S}_{\mathrm{r}}+\mathrm{H} \mathrm{S}_{\mathrm{T}}\right) \mathrm{p}_{\mathrm{a}} \delta \mathrm{p}_{\mathrm{a}} \mathrm{d} \Omega+\int_{\Omega} \mathrm{p}_{\mathrm{a}} \mathrm{n}(\mathrm{H}-1) \dot{S}_{\mathrm{T}} \delta \mathrm{p}_{\mathrm{A}} \mathrm{d} \Omega+$ $\int_{\Omega} p_{a}\left(1-S_{r}+H S_{r}\right) \delta_{j i} \dot{\varepsilon}_{i j} \delta p_{0} d \Omega-\int_{\Omega} d i v\left[p_{a} k_{a} \operatorname{grad}\left(\frac{P_{a}}{\gamma_{a}}+z\right)\right] \delta p_{a} d \Omega-$ $\int_{\Omega} \operatorname{div}\left[\sigma_{\mathrm{a}} H k_{w} \operatorname{grad}\left(\frac{P_{w}}{\gamma_{w}}+z\right)\right] \delta p_{a} d \Omega+\int_{S_{\phi_{a}}} \phi_{a} d S_{\phi_{a}}=0$

Le couplage entre le solide et l'eau est assuré par la présence de la pression d'eau dans la première intégrale et de la variation du volume du sol dans la deuxième intégrale. Le couplage entre le solide et l'air est assuré par la présence de la pression d'air dans la première intégrale et de la variation du volume du sol dans la dernière intégrale. Le couplage entre l'eau et l'air est assuré par la présence du coefficient de solubilité de l'air dans la dernière intégrale.

L'application du principe variationnel choisi à l'analyse du comportement au cours du temps des massifs de sols élastoplastiques non saturés donne le système d'équations suivant, que l'on doit résoudre dans le domaine fixe $\Omega$ :

$$
\begin{aligned}
& \mathrm{dF}=\int_{\Omega} \mathrm{D}_{e} \varepsilon_{j} \delta \varepsilon_{j} \mathrm{~d} \Omega+\int_{\Omega} \gamma_{u}\left(\mathrm{~F}_{e}-1\right) \mathrm{h}_{\mathrm{a}} \delta \varepsilon_{j} \mathrm{~d} \Omega-\int_{\Omega} \gamma_{w} \mathrm{~F}_{e} \mathrm{~h}_{w} \delta \varepsilon_{j} \mathrm{~d} \Omega- \\
& -\int_{\Omega} F_{j} \delta u_{j} \mathrm{~d} \Omega-\int_{S_{\sigma}} T \delta u_{j} d S_{\sigma}-\int_{\Omega}\left(F_{e}-1\right) \gamma_{a} z \delta \varepsilon_{j} d \Omega+ \\
& \int_{\Omega} F_{t} \gamma_{w} z \delta \varepsilon_{y j} d \Omega=0 \\
& \mathrm{dF}_{\mathrm{w}}=\int_{\Omega} \gamma_{w}\left(n g_{1} \mathrm{~m}_{1}^{\top} \mathrm{D}_{\mathrm{e}}+\mathrm{S}_{\mathrm{r}} \delta_{i j}\right) \frac{\partial \varepsilon_{i j}}{\partial \mathrm{t}} \delta \mathrm{h}_{\mathrm{w}} \mathrm{d} \Omega_{-} \\
& \int_{\Omega} n \gamma_{w}^{2}\left(g_{2}+g_{1} F_{e}\right) \frac{\partial h_{w}}{\partial t} \delta h_{w} d \Omega+ \\
& +\int_{\Omega} n \gamma_{w} \gamma_{w}\left(g_{2}+g_{1} F_{e}\right) \frac{\partial h_{b}}{\partial t} \delta h_{w} d \Omega+\int_{\Omega} k_{w} h_{w j} \gamma_{w} \delta h_{w} d \Omega+ \\
& +\int_{S_{v_{w}}} \gamma_{w} \phi_{w} \delta h_{w} d S_{v_{w}}=0 \\
& \mathrm{dF}_{\mathrm{a}}=\int_{\Omega} \gamma_{\mathrm{a}} \mathrm{p}_{\mathrm{u}}\left[\mathrm{n}(\mathrm{H}-1) \mathrm{m}_{1}^{\top} \mathrm{g}_{3} \mathrm{D}_{\mathrm{e}}+\left(1-\mathrm{S}_{\mathrm{r}}+\mathrm{H} \mathrm{S}_{\mathrm{r}}\right)\right] \frac{\partial \varepsilon_{\mathrm{j}}}{\partial t} \delta_{k} \delta \mathrm{h}_{\mathrm{a}} \mathrm{d} \Omega- \\
& -\int_{\Omega} \gamma_{a} \gamma_{w}\left[\rho_{a} n(H-1) g_{1} F_{e}+\rho_{a} n(H-1) g_{2}\right] \frac{\partial h_{w}}{\partial t} d \Omega+
\end{aligned}
$$

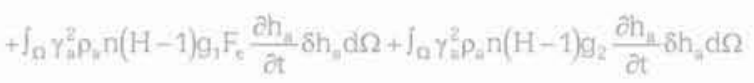

$$
\begin{aligned}
& +\int_{\Omega} \gamma_{\mathrm{a}}^{2} \mathrm{C}_{\mathrm{a}} \mathrm{n}\left(1-\mathrm{S}_{\mathrm{r}}+\mathrm{HS}_{\mathrm{r}}\right) \frac{\partial \mathrm{h}_{\mathrm{a}}}{\partial \mathrm{t}} \delta \mathrm{h}_{\mathrm{a}} \mathrm{d} \Omega+ \\
& +\int_{\Omega} \gamma_{a} \mathrm{k}_{\mathrm{a}} \mathrm{h}_{\mathrm{z}, j} \delta \mathrm{h}_{\mathrm{a}, \mathrm{i}} \mathrm{d} \Omega+\int_{\Omega} \gamma_{\mathrm{a}} H \mathrm{k}_{\mathrm{w}} \mathrm{h}_{\mathrm{w}, \mathrm{j}} \delta \mathrm{h}_{\mathrm{a} . \mathrm{d}} \mathrm{d} \Omega+\int_{\mathrm{Sva}} \gamma_{\mathrm{a}} \phi_{\mathrm{a}} \\
& \delta h_{i} d S_{V_{a}}=0
\end{aligned}
$$

et auquel il faut ajouter les conditions aux limites et les conditions initiales.

Les notations utilisées sont les suivantes :

$\mathrm{dF}$ : fonctionnelle associée aux déformations du squelette :

$\mathrm{dF}_{w}$ : fonctionnelle associée à l'écoulement de l'eau ; $\mathrm{dF}_{\mathrm{a}}$; fonctionnelle associée à l'écoulement de l'air : $\mathrm{D}_{\mathrm{e}}$ : matrice de souplesse du milieu global relative aux variations de $\sigma_{i j}+p_{i} \delta_{i j}$

$\varepsilon_{\mathrm{v}}$ : tenseur des déformations du milieu global ; 
$\gamma_{\mathrm{a}}$ : poids volumique de l'air $\left(\mathrm{kN} / \mathrm{m}^{3}\right)$;

$\gamma_{w}$ : poids volumique de l'eau $\left(\mathrm{kN} / \mathrm{m}^{3}\right)$;

$\mathrm{F}_{\mathrm{e}}=\mathrm{D}_{e} \mathrm{D}_{\mathrm{s}}$;

$D_{s}$ : matrice de souplesse du milieu global relative aux variations de $p_{\mathrm{p}}-\mathrm{p}_{\mathrm{w}}$;

$\mathrm{h}_{\mathrm{a}}$ : charge d'air $(\mathrm{m})$;

$\mathrm{h}_{w}$ : charge d'eau (m);

$\mathrm{F}$ : forces volumiques $\left(\mathrm{kN} / \mathrm{m}^{3}\right)$;

$\mathrm{T}$ : forces surfaciques $(\mathrm{kPa})$;

$\mathrm{z}$ : cote du point courant ( $\mathrm{m}$ )

$\rho_{\mathrm{i}}$ : masse volumique de l'air $\left(\mathrm{kg} / \mathrm{m}^{3}\right)$;

$\mathrm{n}$ : porosité du sol :

$\mathrm{H}$ : coefficient de Henry $(\mathrm{H}=0,02)$;

$m_{1}^{\top}=[010]$;

$g_{1}$ : dérivée de la fonction de saturation par rapport à $\sigma_{i j}+p_{\mathrm{z}} \delta\left(\mathrm{kPa}^{-1}\right)$

$\mathrm{g}_{2}$ : dérivée de la fonction de saturation par rapport à

$\mathrm{p}_{\mathrm{a}}-\mathrm{p}_{\mathrm{w}}\left(\mathrm{kPa}^{-1}\right)$;

$\mathrm{S}_{\mathrm{r}}$ : degré de saturation:

$\mathrm{C}_{\mathrm{a}}$ : compressibilité de l'air $\left(\mathrm{KPa}^{-1}\right)$;

$\mathrm{k}_{\mathrm{a}}$; coefficient de perméabilité à l'air $(\mathrm{m} / \mathrm{s})$;

$\mathrm{k}_{\mathrm{w}}$ : coefficient de perméabilité à l'eau $(\mathrm{m} / \mathrm{s})$.

\section{8}

\section{Méthode de résolution numérique}

L'application du principe variationnel aux équations et la discrétisation dans l'espace (éléments finis) et dans le temps (méthode implicite) conduisent au système matriciel suivant :

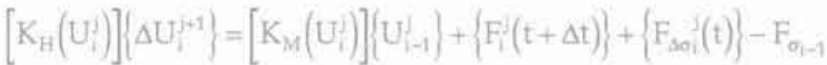

avec:

$$
\begin{aligned}
& K_{H}=\left[\begin{array}{ccc}
R & -C_{u w} & C_{w a} \\
C_{w a} & -C_{w w}+\Delta t K_{w} & C_{w a} \\
C_{a u} & -C_{a w}+\Delta t K_{w a} & E_{a}+\Delta t K_{u}
\end{array}\right] \quad \Delta U=\left\{\begin{array}{l}
\Delta U \\
\Delta H_{w} \\
\Delta H_{a}
\end{array}\right\} \\
& K_{M}=\left[\begin{array}{ccc}
0 & C_{u w} & -C_{u a t} \\
0 & -\Delta t K_{w} & 0 \\
0 & -\Delta t K_{w a} & -\Delta t K_{a}
\end{array}\right] \quad U=\left\{\begin{array}{l}
U \\
H_{w w} \\
H_{a}
\end{array}\right\} \\
& F(t+\Delta t)=\left\{\begin{array}{l}
F(t+\Delta t) \\
Q_{w}(t+\Delta t) \\
Q_{a}(t+\Delta t)
\end{array}\right\} \quad F_{\sigma}(t)=\left\{\begin{array}{l}
F_{\sigma}(t) \\
0 \\
0
\end{array}\right\} \quad F_{\Delta \sigma}(t)=\left\{\begin{array}{l}
F_{\Delta \sigma}(t) \\
0 \\
0
\end{array}\right\}
\end{aligned}
$$

Dans la méthode de résolution décrite icí, le schéma numérique implicite, plus stable numériquement, a été choisi en raison du caractère non linéaire du problème.

La résolution du problème est réalisée par un procédé itératif. A chaque itération on cherche les valeurs des inconnues principales, des inconnues secondaires. du résidu et du paramètre d'écrouissage de telle façon que, pour j (nombre d'itérations) tendant vers l'infini. les champs de valeurs des inconnues soient solution du problème complet. Si l'on admet la solution connue à l'itération $j-1$, la détermination des inconnues principales, des inconnues secondaires, du résidu et des paramètres d'écrouissage s'effectue en deux étapes.

\section{Première étape}

On détermine les inconnues principales (déplacements u et v, charges d'eau et d'air) et secondaires (contraintes et vitesses d'écoulement de l'eau et de l'air) solutions du problème linéaire statiquement et cinématiquement admissibles, de sorte que les contraintes et les déformations soient reliées par la loi de comportement élastique linéaire.

\section{9}

\section{Deuxième étape}

Le problème est ici non linéaire. A partir des inconnues principales et secondaires statiquement et cinématiquement admissibles déterminées précédemment, on détermine les déplacements, les contraintes, le résidu et les forces d'écrouissage de façon que la contrainte généralisée asșociée et la force d'écrouissage soient plastiquement admissibles, c'est-à-dire telles que $\mathrm{f}(\sigma, \mathrm{k}) \leq 0$, et que les incréments des contraintes et des pressions soient statiquement admissibles.

\section{9}

\section{Exemples de calculs}

\section{1}

\section{Consolidation élastoplastique en milieu non saturé}

Ce premier exemple porte sur la modélisation axisymétrique d'un tube épais, de rayon intérieur $\mathrm{r}=2 \mathrm{~m}$ et de rayon extérieur $r_{\mathrm{f}}=12 \mathrm{~m}$. Le maillage et les conditions aux limites sont représentés sur la figure 1 (déplacements radiaux uniquement; surface externe imperméable et immobile; surface interne perméable avec des charges d'air et d'eau constantes). Une pression uniformément répartie de $100 \mathrm{kPa}$ est appliquée à l'intérieur du tube. Dans cet exemple, le sol est supposé élastoplastique et représenté par le modèle d'Alonso et al. (1990), Les données numériques utilisées pour cet exemple sont rassemblées dans le tableau I (exemple 1).

Les figures 2, 3, 4 et 5 présentent respectivement l'évolution des déplacements radiaux, des déformations plastiques totales, des charges d'eau et des charges d'air suivant la coupe $A B$ (Fig. 1)

Les résultats de ce calcul sont conformes à ce qu'une analyse physique du problème suggère : une

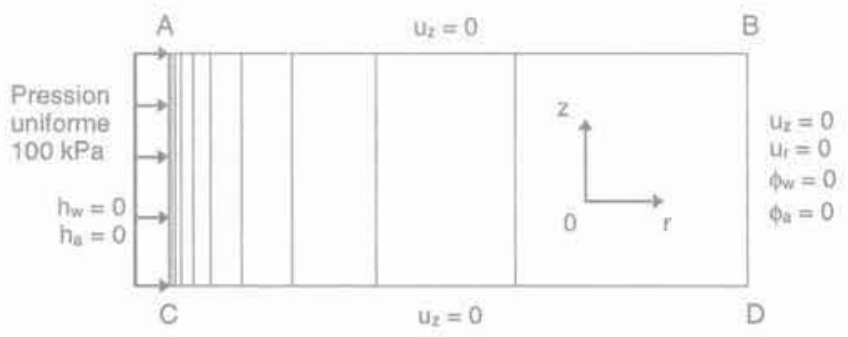

FG. 1 Maillage et conditions aux limites (premier exemple).

FE Mesh and boundary conditions (first example). 
TAALEAU1 Valeurs numériques des paramètres de calculs. Numerical values of the parameters.

\begin{tabular}{|c|c|c|}
\hline Paramètre & Exemple 1 & Exemple 2 \\
\hline Poids volumique du sol $\gamma\left(\mathrm{kN} / \mathrm{m}^{3}\right)$ & 20 & 0 \\
\hline Module d'Young E (kPa) & 10000 & 9600 \\
\hline Coefficient de Poisson v & 0,2 & 0,4 \\
\hline Module de déformation volumique en succion $\left(\mathrm{kPa}^{-1}\right)$ & 18000 & 54500 \\
\hline Indice des vides initial $e_{\circ}$ & 0,34 & 0,9 \\
\hline Coefficient de perméabilité à l'eau du sol saturé $k_{\text {wisst }}(\mathrm{m} / \mathrm{s})$ & $10^{-8}$ & $6.10^{-10}$ \\
\hline Constante du degré de saturation a, & 1 & 1 \\
\hline Constante du degré de saturation $\mathrm{b}_{s}\left(\mathrm{KPa}^{-1}\right)$ & $-0,0001$ & $-0,00001$ \\
\hline Constante du degré de saturation $\mathrm{c}_{\mathrm{n}}\left(\mathrm{KPa}^{-1}\right)$ & 0,012 & $5.10^{-4}$ \\
\hline Degré de saturation résiduel $S_{r u}$ & 0 & 0 \\
\hline Constante pour la perméabilité à l'eau $\alpha$ & 3 & 3 \\
\hline Constante pour la perméabilité à l'air b & $1,8.10^{-10}$ & $0,41 \cdot 10^{-5}$ \\
\hline Constante pour la perméabilité à l'air c & 1 & 3 \\
\hline Contrainte verticale $\sigma_{\mathrm{y}}(\mathrm{kPa})$ & 200 & 200 \\
\hline Poids volumique de l'eau $\gamma_{w}\left(\mathrm{kN} / \mathrm{m}^{3}\right)$ & 10 & 10 \\
\hline Poids volumique de lair $\gamma_{a}\left(\mathrm{kN} / \mathrm{m}^{3}\right)$ & 0,012 & 0,012 \\
\hline Coefficient de Henry H & 0,02 & 0,02 \\
\hline Coefficient de compressibilité de l'air $\mathrm{C}_{i}\left(\mathrm{kPa}^{-1}\right)$ & 1,2. $10^{-5}$ & $1,2.10^{-5}$ \\
\hline Pente de la courbe vierge $\lambda$ & 0,014 & \\
\hline Pente des courbes de déchargement-rechargement $x$ & 0,024 & \\
\hline Paramètre dẻfinissant la rigiditẻ du sol r & 0,82 & \\
\hline Paramètre contrôlant l'augmentation de la rigidité du sol avec la succion $\beta\left(\mathrm{kPa}^{-1}\right)$ & 16400 & \\
\hline Pression de préconsolidation initiale $\mathrm{P}_{\mathrm{co}}(\mathrm{kPa})$ & 100 & \\
\hline Contrainte de référence $\mathrm{P}_{r}(\mathrm{kPa})$ & 100 & \\
\hline Coefficient contrôlant l'augmentation de la cohésion avec la succion $\mathrm{P}_{\text {ii }}$ & 0,02 & \\
\hline Pente de la courbe d'état critique M & 1,24 & \\
\hline
\end{tabular}

Déplacement radial $(\mathrm{m})$

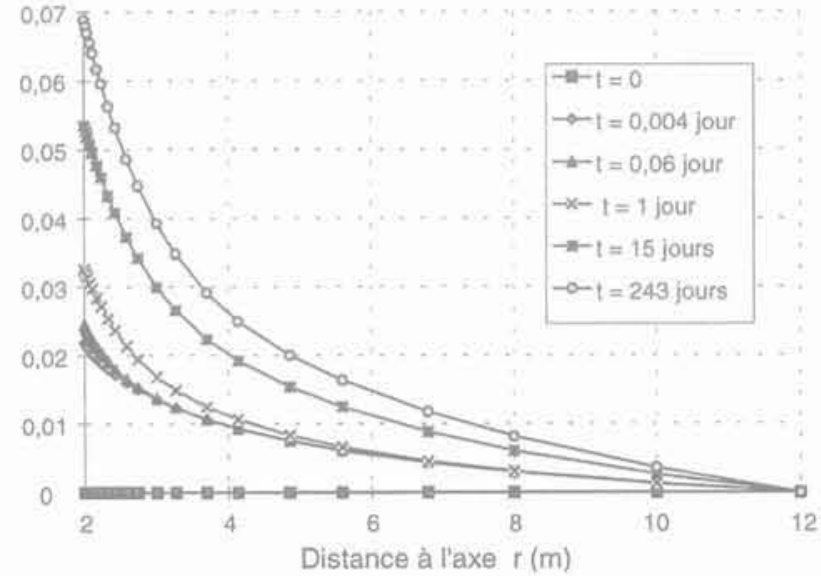

FIG.2 Variations des déplacements radiaux en fonction du temps.

Variations with time of the radical displacements.

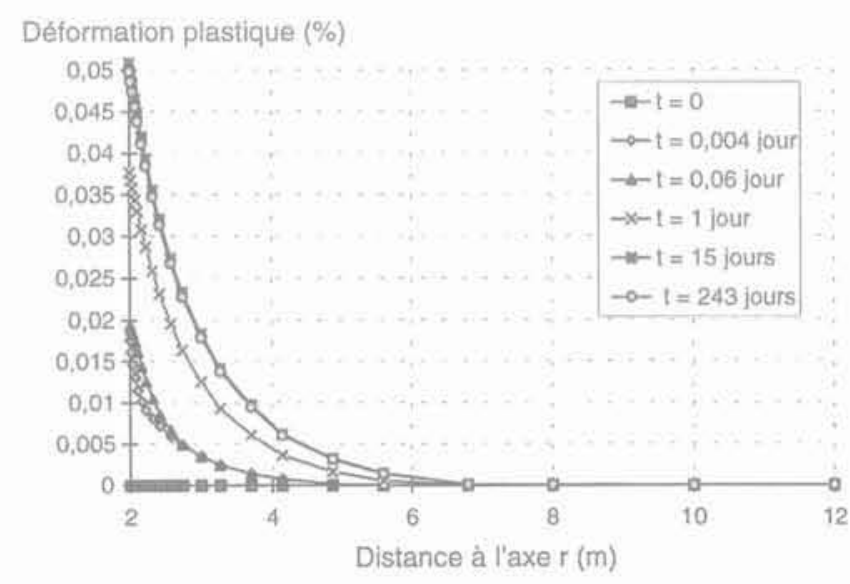

FG. 3 Variations des déformations plastiques en fonction du temps.

Variations with time of the plastic strains. 


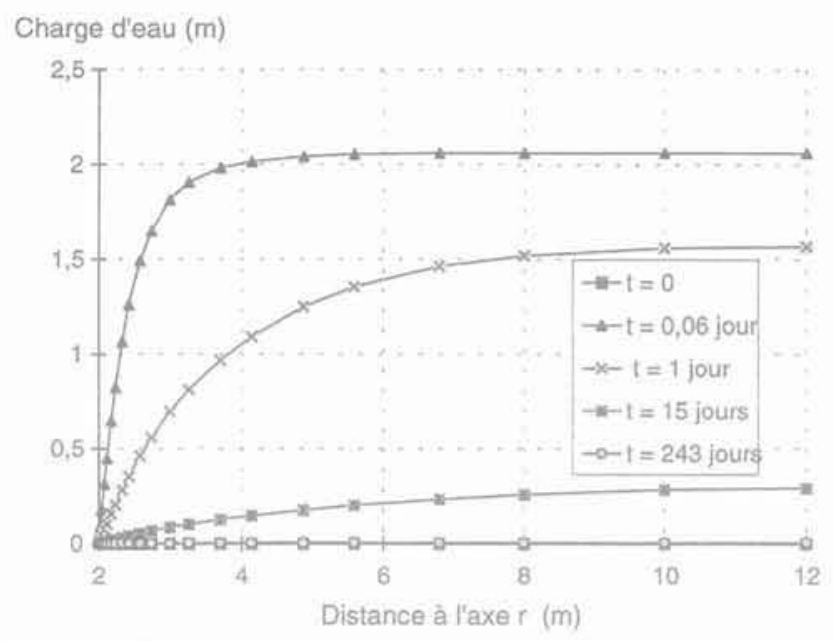

Fig. 4 Variations des charges d'eau en fonction du temps.

Variations with time of the water head.

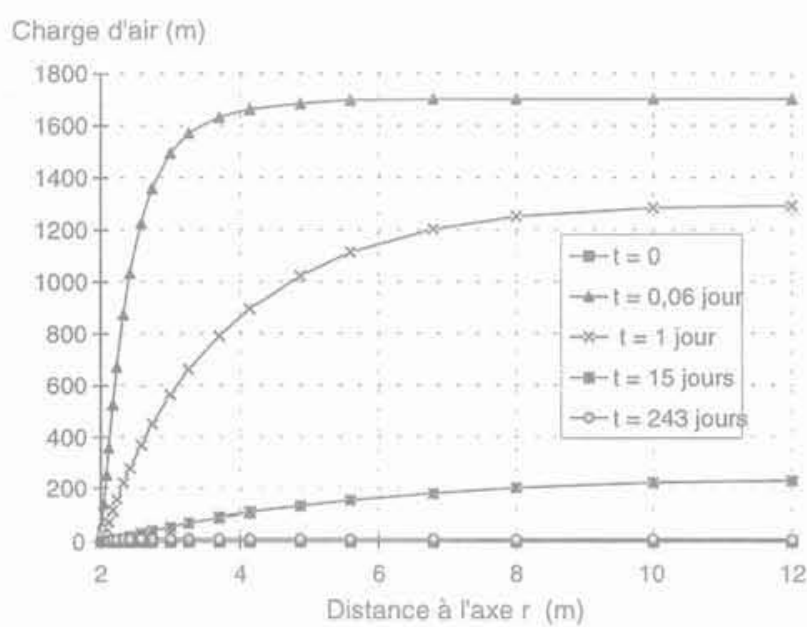

FiG.5 Variations des charges d'air en fonction du temps.

Variations with time of the air head.

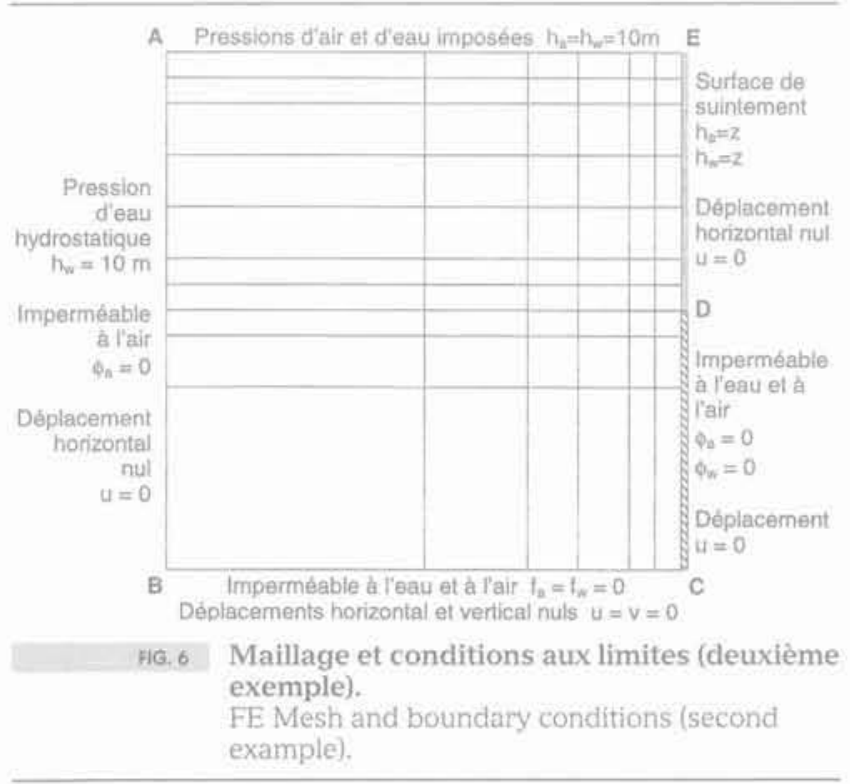

augmentation instantanée des charges d'eau et d'air au départ, puis leur diminution au cours du temps, qui traduit le processus de consolidation lié au drainage des deux fluides ; les déformations plastiques et les déplacements radiaux augmentent au cours du temps, en partant de la paroi intérieure du tube, ce qui traduit respectivement la plastification progressive du matériau et le transfert des sollicitations appliquées des fluides vers le squelette du sol.

\section{2}

\section{Diffusion couplée en milieu déformable}

Nous avons effectué un calcul bidimensionnel qui permet de tester les conditions d'écoulements des deux fluides (eau et air). Ce test a été effectué sur un carré de $10 \mathrm{~m}$ de côté, dont les conditions aux limites sont repré. sentées sur la figure 6. Le côté $\mathrm{AB}$ est soumis à des conditions de charge d'eau imposée, égale à $10 \mathrm{~m}$ (le repère des altitudes est placé à la base du maillage), et de flux d'air nul; sur la surface AE du massif, la pression d'eau et la pression d'air sont nulles (charge constante égale à 10 $\mathrm{m})$; le còté DE est soumis à des conditions de surface de suintement (charges d'eau et d'air égales à la cote du point courant) et, enfin les côtés BC et CD sont soumis à des conditions de flux nul pour les deux phases. Le so est considéré comme élastique linéaire et aucun chargement mécanique n'est appliqué. Les paramètres du calcul sont donnés dans le tableau I (exemple 2).

Les conditions initiales du calcul sont que les charges d'eau et d'air sont égales à $10 \mathrm{~m}$ et que les déplacements verticaux et horizontaux sont nuls.

Ce problème correspond donc au drainage par une tranchée maintenue vide (pressions d'air et pressions d'eau nulles sur ED) d'une couche de sol non saturé initialement en équilibre hydrostatique, avec des pressions d'eau et d'air nulles sur la surface AE, deux surfaces imperméables à l'eau et à l'air (BC, CD) et une surface $\mathrm{AB}$ imperméable à l'aír mais maintenue en équilibre hydrostatique pour l'eau.

Pour illustrer les résultats de ce calcul, nous avons représenté sur les figures 7 à 10 les équipotentielles et les lignes de courant pour l'eau et pour l'air à deux instants : $t=24000$ s et $t=2698000 \mathrm{~s}$. La figure 11 montre pour sa part l'allure des déplacements verticaux à l'instant $\mathrm{t}=24000 \mathrm{~s}$.

Les écoulements de l'eau et de l'air dans le sol évoluent séparément en fonction de leurs propres conditions aux limites. Pour comparer les distributions de charges d'eau et d'air dans le sol aux résultats connus des calculs d'écoulements permanents, nous avons effectué, avec les mêmes conditions aux limites, des calculs d'écoulements dans lesquels le massif est supposé homogène, avec, d'une part, les conditions aux limites de l'écoulement de l'eau et, d'autre part, les conditions aux limites de l'écoulement de l'air. Les résultats sont représentés, respectivement, sur les figures 12 et 13 . On observe que l'état des écoulements à l'instant $t=2698$ 000 s est pratiquement identique à l'écoulement permanent de chacune des phases en milieu homogène saturé. Cette observation traduit la faiblesse de l'influence des conditions d'interaction des phases en milieu non saturé dans les conditions de ce calcul.

\section{Conclusion}

Cet article a décrit de façon sommaire les équations qui nous ont servi à développer un modèle numérique pour l'analyse du comportement couplé des massifs élastoplastiques non saturés. Ce modèle a été implanté dans le code de calcul aux éléments finis CESAR-LCPC. 


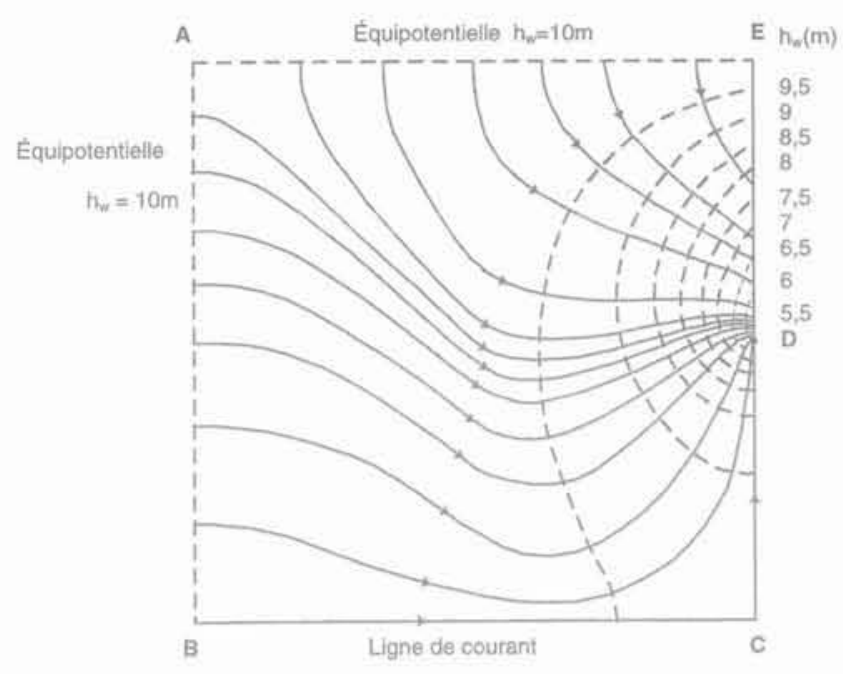

FIG. 7 Équipotentielles et lignes de courant de l'eau au temps $\mathrm{t}=24000 \mathrm{~s}$ (environ $6,7 \mathrm{~h}$ ): $\mathrm{AB}, \mathrm{AE}$ - équipotentielles; $\mathrm{BC}, \mathrm{CD}$ - lignes de courant ; ED - surface de suintement. Equipotentials and flow lines for water at time $\mathrm{t}=24,000$ s (approximately $6.7 \mathrm{~h}$ ): $\mathrm{AB}, \mathrm{AE}-$ equipotentials: $\mathrm{BC}, \mathrm{CD}$ - flow lines : $\mathrm{ED}$ seepage surface.

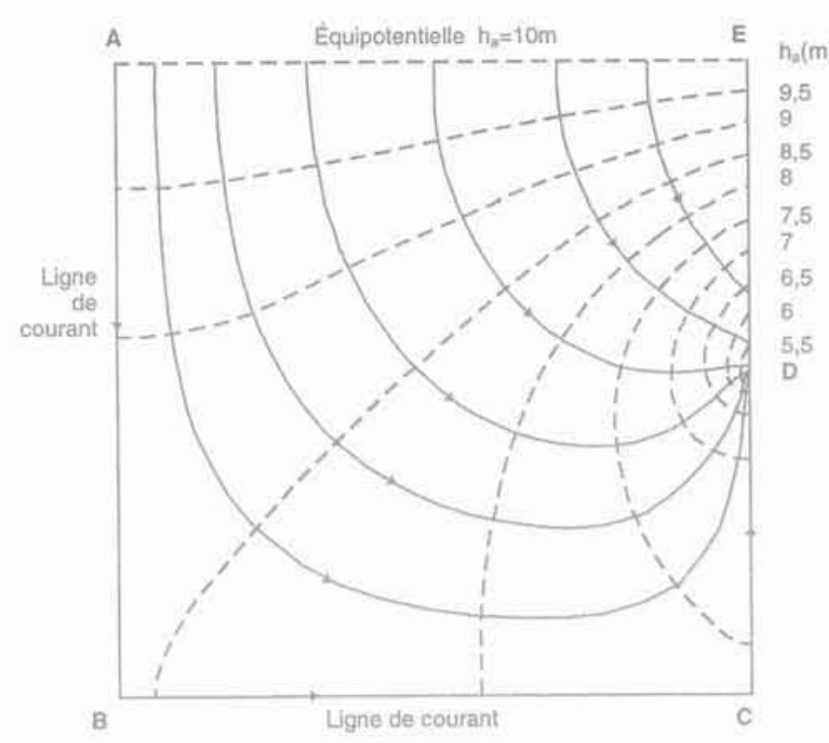

FIG. 8 Équipotentielles et lignes de courant de l'air au temps $t=24000 \mathrm{~s}: \mathrm{AE}$ équipotentielle: $A B, C D, C E$ - lignes de courant; ED - surface de suintement. Equipotentials and flow lines for air at time $t=24,000$ s: $A E$ - equipotentials; $A B, C D, C E-$ flow lines: ED - seepage surface.

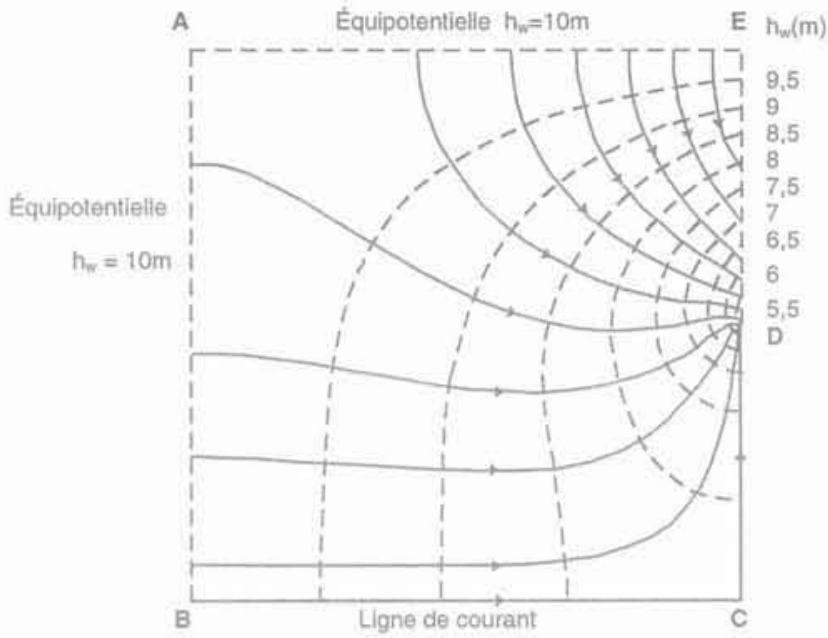

FG.9 Equipotentielles et lignes de courant de l'eau au temps $t=2698000 \mathrm{~s}$ (environ $31 \mathrm{j}$ ): $\mathrm{AB}, \mathrm{AE}$ - équipotentielles; $\mathrm{BC}, \mathrm{CD}$ - lignes de courant ; ED - surface de suintement. Equipotentials and flow lines for water at time $\mathrm{t}=2,698,000$ s (approximataly 31 days): $\mathrm{AB}, \mathrm{AE}$ - equipotentials; $B C, C D$ - flow lines: $E D$ seepage surface.

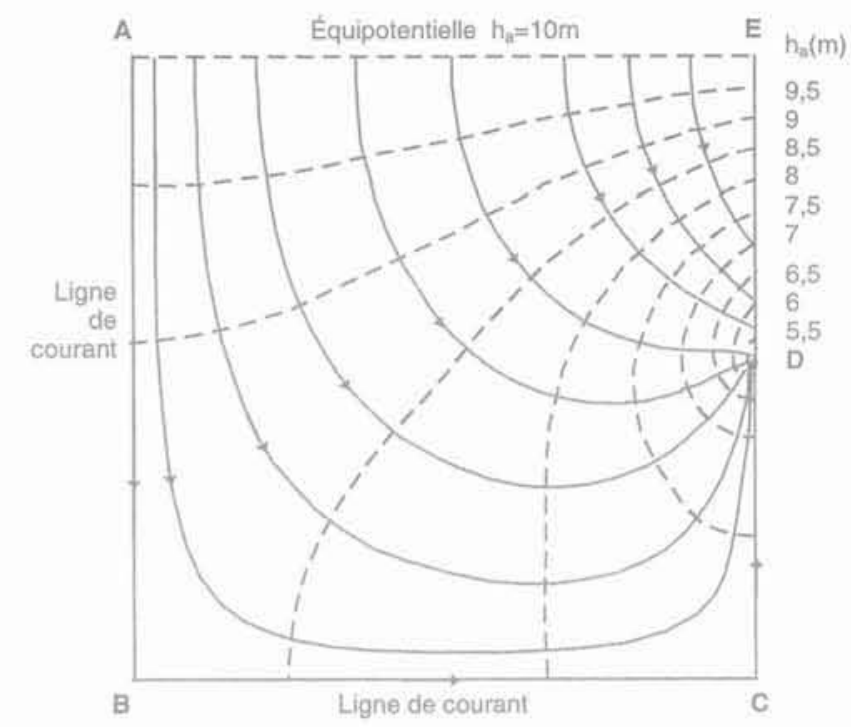

FIG. 10 Équipotentielle et lignes de courant de l'air au temps $\mathrm{t}=2698000 \mathrm{~s}: \mathrm{AE}$ équipotentielle; $\mathrm{AB}, \mathrm{CD}, \mathrm{CE}-$ lignes de courant; ED - surface de suintement. Equipotentials and flow lines for air at time $t=2,698,000$ s: $A E$ - equipotential, $A B, C D, C E$ - flow lines: ED - seepage surface.
Les tests effectués dans les cas uni- et bidimensionnels et sur deux géométries différentes (plane et axisymétrique) ont montré les aptitudes de ce modèle à reproduire de façon satisfaisante les conditions des tests proches de l'état de ces matériaux in situ. Ses développements passent par l'amélioration des équations élémentaires, pour assurer une bonne représentativité des résultats des calculs. 
A

Soulèvement

\begin{tabular}{|l|}
\hline $7 \mathrm{~cm}$ \\
\hline \\
\hline $6 \mathrm{~cm}$ \\
\hline $5 \mathrm{~cm}$ \\
\hline $4 \mathrm{~cm}$ \\
\hline $3 \mathrm{~cm}$ \\
\hline $2 \mathrm{~cm}$ \\
\hline $1 \mathrm{~cm}$ \\
\hline $0 \mathrm{~cm}$ \\
\hline
\end{tabular}

E

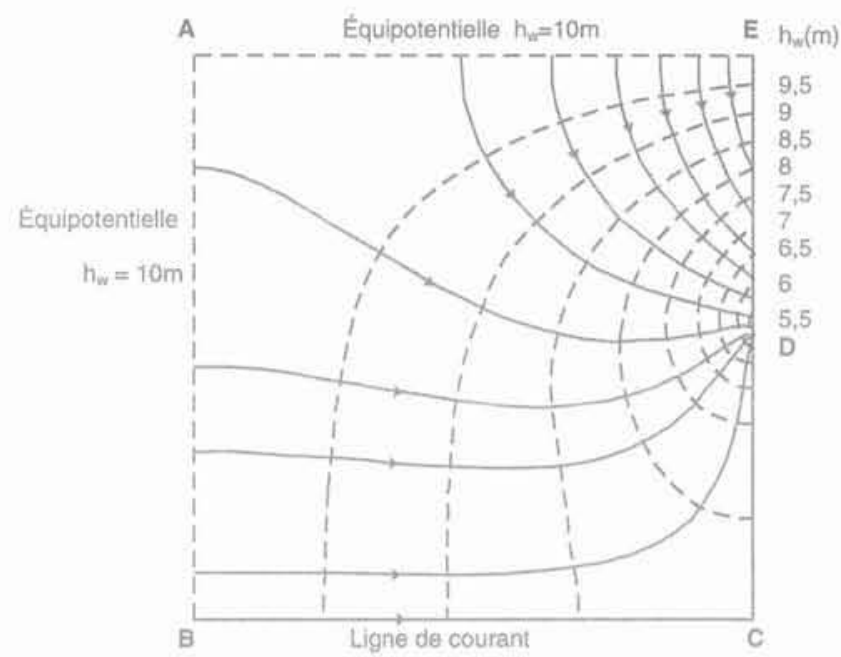

FG. 12 Équipotentielles et lignes de courant pour un écoulement permanent dans les conditions définies pour l'écoulement de l'eau clans le massif: $A B$. AE équipotentielles; BC, CD - lignes de courant; ED - surface de suintement. Equipotentials and flow lines for steady seepage of water with the same boundary conditions as in Fig. 7 and 9 .

gonflement) au temps $\mathrm{t}=24000 \mathrm{~s}$.

Lines of equal vertical displacements (heave) at time $\mathrm{t}=24,000 \mathrm{~s}$.

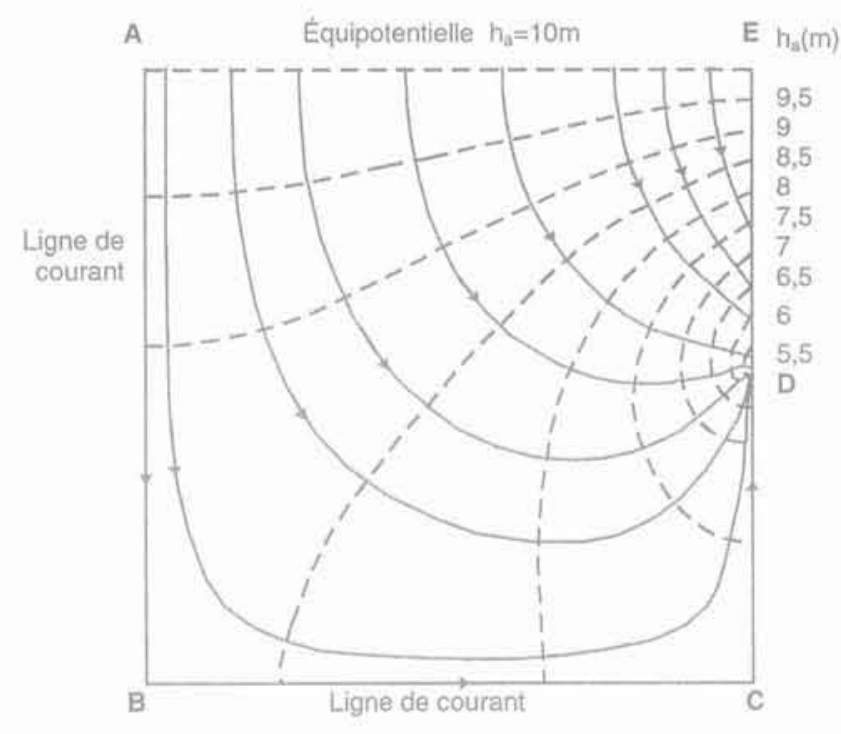

FIG.13 Équipotentielles et lignes de courant pour un écoulement permanent dans les conditions définies pour l'écoulement de l'air dans le massif: $\mathrm{AE}$ - équipotentielle : $A B, B C, C D$ - lignes de courant; ED surface de suintement.

Equipotentials and flow lines for steday seepage of air with the same boundary conditions as in Fig. 8 and 10.

\section{Bibliographie}

Abida H. - "La modélisation dés sols non saturès - Analyse numérique $»$. Thèse de doctorat, École nationale des ponts et chaussées, Paris, 1992, 271 p.
Alonso A.E., Batlle F., Gens A., Lloret A. « Consolidation analysis of partially saturated soils. Application to earthdam construction 1). Proceedings, 6th Inter- national Conference on Numerical Methods in Geomechanics, Innsbruck,

Balkema, 1988 , p. 1303-1308.

Alonso E.E., Gens A. Hight D.W. - $\alpha$ Spe- 
cial problems soils - General Report (session 5) v. Proceedings, 9th European Conference on Soil Mechanics and Foundation Engineering. Dublin, vol. 5, 1987, p. 1-60.

Alonso A.E., Gens A., Josa A. - « A constitutive model for partially saturated soils n. Géotechnique 40, n³, 1990. p. 405-430.

Alonso E.E., Gens A., Lloret A. - " Double structure model for the prediction of long-term movernents in expansive materials in. Computer Methods and Advances in Geomechanics, Rotterdam. Balkema, 1991, p. 541-548.

Biarez J., Fleureau J.M, Zerhouni M.I., Soepandji B.S. - "Variations de volume des sols argileux lors des cycles de drainage-humidification 1 . Revue française de géotechnique, $n^{\circ} 41,1988$, p. 68-71.

Bishop A.W. Blight G.E. - $\approx$ Some aspects of effective stress in saturated and partly saturated soils 1. Géotechnique, vol. 13 . n० 3. 1963, p. 177-197.

Bishop A.W.. Donald I.B. - " The experimental study of partly saturated soils n. Géotechnique, vol. $13, n^{\circ} 3,1961$, p. 177-197.

Fredlund D.G. - $\propto$ Discussion leader's report x. Proceedings, 12th International Conference on Soil Mechanics and Foundation Engineering, Rio de Janeiro, vol. 5,1989 , p. 2861-2878.

Fredlund D.G., Morgenstern N.R. «Constitutive relations for volume change in unsaturated soils n., Canadian Geotechnical Journal, n 13, 1976, p. 261276.

Gatmiri B., Tavakoli S., Moussavi S. Delage P. - « Numerical approach of elastoplastic consolidation of unsatura. ted soils x. Proceedings of the first Inter- national Conference on Unsaturated Solls, /UNSAT'95 Paris/, France, vol. 2. 1995, p. 1057-1064.

Gens A., Vaunat J., Ledesma A. - « Analy sis of hydratation of an engineered barrier in a radioactive waste repository scheme using an elastoplastic model $x$. Proceedings of the first International Conference on Unsaturated Soils, /UNSAT 95 Paris/, France, vol. 2, 1995, p. 1057-1064

Hayes L.J. - - "Finite element patch approximations and alternating direcfions methods 1. . Mathematics and Computer in Simulation, XXII, 1980, p. 25-29.

Haves L.J. - « A modified backward time discretization for nonlinear parabolic equations using patch approximations $x$. SIAM J. Numer. Anal., vol. 18, n 5, 1981, p. $781-793$.

Jennings J.E.B., Burland J.B. - "Limitations to the use of effective stresses in partly saturated soils is. Géotechnique, $12, n^{\circ} 2,1962$, p. $125-144$.

Karube D. Kato S. - " Yield functions of unsaturated soil 9 . Proceedings, 12th International Conference on Soil Mechanics and Foundation Engineering, Rio de Janeiro, vol, 1, 1989, p. 615618.

Kohgo Y., Nakano M., Miyazaki T. - $\alpha$ Theoretical aspects of constitutive modelling for unsaturated soils n. Soils and Foundations, vol. $33, n^{\circ} 4,1991$, p. $49-63$.

Musy A - - Simulation des écoulements en milieu poreux non saturé n. Journa! of Hycirology, $n^{\circ} 29,1976, \mathrm{p}, 1-21$.

Nanda $A_{i}-$ « Finite element analysis of unsaturated coupled flow and deforma-

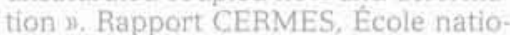
nale des ponts et chaussées, 1989, $18 \mathrm{p}$.
Narasimhan T.N., Neuman S.P., Witherspoon P.A. - « Finite element method for subsurface hydrology using a mixed explicit-implicit scheme $x$. Water Resources Research, vol. 14, n ${ }^{\circ}$ 5, 1978, p. 863-877.

Narasimhan T.N., Witherspoon P.A. «Numerical model for saturated-unsaturated flow in deformable porous media. "Theory" „, Water Resources Research, vol. 13, n³, 1977, p. 657-664.

Narasimhan T.N., Witherspoon P.A. uNumerical model for saturated-unsaturated flow in deformable porous media. "Algorithm" w. Water Resources Research, vol. 14, n². 2. 1978, p. 255-261.

Narasimhan T.N., Witherspoon P.A. a Numerical model for saturated-unsaturated flow in deformable porous media. "Applications" in. Water Resources Research, vol. 14, n ${ }^{6} 6,1978$, p. 1017-1034.

Shrefler B.A., Zhan Xiaoyong, - " A fully coupled model for water flow and airflow in deformable porous medía $n$. Water Resources Research, vol. $29, \mathrm{n}^{\circ} 1$. 1993, p. 155-167.

Matyas E.L, Radhakrishnan A.S. "Volume change characteristics of partially saturated soils n. Géotechnique, vol. 18, 1968, p. 432-448.

Touma J. Vauclin M. - $\pi$ Experimental and numerical analysis of two-phase infiltration in a partially saturated soll $x$. Transport in Porous Media n $n^{\circ} 1,1986$, p. $27-55$

Wone E.M. - " Contribution à la modélisation du comportement hydrique et mécanique des remblais routiers en matériaux fins n. Thèse de doctorat, École nationale des ponts et chaussées. Paris, 1995, $210 \mathrm{p}$. 